\title{
Development of an Analytically Described Pitch Regulator for a Wind Turbine to Be Used for Grid Disturbance Studies
}

\author{
Pinar Tokat, Torbjörn Thiringer, and Peiyuan Chen \\ Division of Electric Power Engineering, Department of Energy and Environment, Chalmers University of Technology, \\ 41258 Gothenburg, Sweden \\ Correspondence should be addressed to Pinar Tokat; tokat@chalmers.se
}

Received 19 February 2013; Accepted 7 March 2013

Academic Editor: Onder Ozgener

Copyright (C) 2013 Pinar Tokat et al. This is an open access article distributed under the Creative Commons Attribution License, which permits unrestricted use, distribution, and reproduction in any medium, provided the original work is properly cited.

\begin{abstract}
In this paper, a pitch controller for a variable-speed wind turbine to be used in the high wind speed region is derived. The pitch regulator parameters are determined using analytical expressions and are compared with numerical calculations. In order to derive the pitch regulated wind turbine model, blade element momentum theory is utilized and reformulated analytically. Appropriate simplifications are made and, finally, the analytically derived pitch regulated wind turbine model is tested under grid disturbances such as voltage dips and spinning reserve provision. From this work it was found that by linearizing the blade profiles, one can analytically derive a fully functioned pitch regulator. In spite of all nonlinearities, a single pitch controller setting which is valid for the whole operation region is shown to be sufficient. This system is tested under grid disturbances and it is proven that the system is capable of operating well during a $0 \%$ remaining voltage dip and also during the voltage recovery back to the rated voltage level. Accordingly, grid codes commonly referred to can be handled with this simply derived pitch regulator. Moreover, it is shown that the derived system works well for a spinning reserve application using a $90 \%$ spinning reserve ability and still maintains a robust turbine control.
\end{abstract}

\section{Introduction}

Due to the increasing concern about the emissions caused by the usage of fossil fuels and the security issues regarding nuclear power, wind energy has nowadays become a popular source of electric power production. Wind energy is one of the fastest growing energy sources, which means that a great number of wind power plants have been installed and many more are to be installed further. Accordingly, the impact of wind energy units on the grid is of utmost importance to be studied.

Many wind turbine studies require modeling and simulation of wind turbines. Today, most wind turbines are of the variable-speed type and pitching is an essential feature of the variable-speed operation. In [1], pitch controller is explained but not derived, and in [2] pitch regulator is derived using a fairly extensive procedure. In $[3,4]$, the pitch regulator is designed using complex control methods. However, a simple design of a pitch regulator based on an analytical wind turbine model is not found in the literature. Such a "simple and analytically described" pitch regulator design would be useful while performing and in particular while comparing different studies.

Motivated by the reasons above, the purpose of this paper is to provide a fully traceable and analytically expressed derivation of a pitch regulator for a power system generic wind turbine, so that it can be used for complex studies. One of the goals of this work is to present the required steps such as modifying the blade element momentum theory and linearizing the blade profile of the wind turbine. Furthermore, a target is to demonstrate that the turbine through the pitching activity can handle a voltage dip without the need for a braking chopper. Finally, another aim is to test the system under load balancing disturbances, that is, to demonstrate a spinning reserve application.

\section{Noniterative Blade Element Momentum Theory}

A wind turbine is a device that converts the energy in the wind to electrical energy. The main idea in this conversion 
is to produce a driving torque by the turbine blades, in order to create a torque on the electric generator. For this study, the production of the driving torque is expressed using the blade element momentum theory (BEM) [5].

The rated power of the wind turbine used in this work is $2 \mathrm{MW}$ and the rated rotor speed is $19 \mathrm{rpm}$. The blades of the wind turbine are nonuniformly shaped and have a length of approximately 35 meters for this case. Detailed information about the blade geometry can be found in [6].

Due to the fact that the torque formation is not uniformly distributed along the blade, the turbine blade is divided into smaller pieces during the analysis, where the wind is considered to be homogenous on each piece. These pieces are called the blade segments and on each blade segment the effect of the wind is different.

The blade segment is subjected to two forces created by the wind. These are called the edge and the flap forces; their directions are axial and tangential to the rotor plane, respectively. The edge force, $F_{\text {edge }}$, is the source of the rotation, hence the source of the output torque. The torque produced by each blade segment is called the torque contribution $(\Delta T)$ of the respective segment. The relation between $F_{\text {edge }}$ and $\Delta T$ is

$$
\begin{gathered}
F_{\text {edge }}=\frac{1}{2} \rho W^{2} c \Delta r\left(c_{L} \sin \phi-c_{D} \cos \phi\right), \\
\Delta T=F_{\text {edge }} r=\frac{1}{2} \rho W^{2} c \Delta r\left(c_{L} \sin \phi-c_{D} \cos \phi\right) r,
\end{gathered}
$$

where $\rho$ is the air density, and $W$ is the relative velocity composed by the incoming wind speed and the wind speed due to the rotation of the turbine. $c$ is the chord, and $\Delta r$ is the radial length of the blade segment. $c_{L}$ and $c_{D}$ represent the lift and drag coefficients, respectively, and $\phi$ is the relative flow angle.

The summation of the torque contribution of each segment determines the torque output of one turbine blade. The turbine used in this study has three turbine blades. Therefore, the total output torque of the wind turbine is

$$
T=n_{b} \sum_{i=1}^{m} \Delta T_{i}
$$

Here, $T$ indicates the output torque of the wind turbine, $n_{b}$ is the number of blades, and $m$ is the number of blade segments.

A key point of the BEM for this work is the derivation of the relative flow angle, $\phi$. It is given as

$$
\phi=\tan ^{-1} \frac{\nu_{\omega}\left(1+a^{\prime}\right)}{r \omega_{r}(1-a)},
$$

where $\omega_{r}$ is the angular rotor speed and $r$ is the radius of the segment [7]. $a$ and $a^{\prime}$ are called axial and tangential induction factors, respectively [8]. The problematic point about the $\phi$ calculation is that $a$ and $a^{\prime}$ are calculated iteratively. This, in principle, makes it very difficult to derive an analytical model of the turbine blades and complicates the simulations. However, these factors are of less importance in the high wind

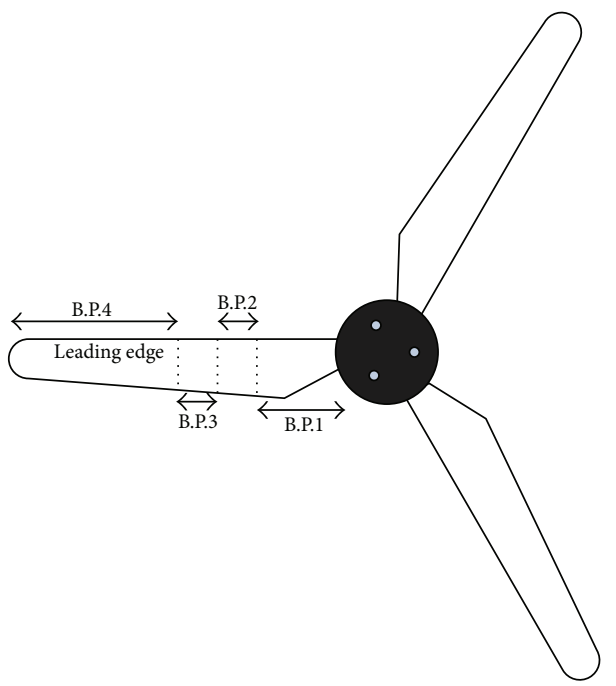

FIgURE 1: The blade profile distribution along the wind turbine blade.

speed region. Therefore, in the high speed region, $\phi$ can be simplified to

$$
\phi=\tan ^{-1} \frac{\nu_{\omega}}{r \omega_{r}}
$$

where $a$ and $a^{\prime}$ are neglected. For more detailed information about the effects of $a$ and $a^{\prime}$, please refer to [6]. Using this expression for $\phi$, the BEM becomes a noniterative method. This is essential for the upcoming derivation of the pitch regulator in analytical terms.

\section{Linearizing the Blade Profile}

The wind acting on the blade has different directions according to the position of the blade. The angle between the incoming wind and the chord line of the turbine blade is called the angle of attack. The angle of attack is an important parameter of the wind turbine, since the lift and drag coefficients are depending on it. Initially, for each angle of attack, there is a set of $c_{L}$ and $c_{D}$. The table which shows the lift and drag coefficient values for different angle of attacks gives the blade profile, as shown in Figure 3. Unfortunately, the relation between these coefficients and the angle of attack is non-linear. It would be convenient to have a linear relation between them in order to build the analytical model.

Due to the nonuniform shape of the turbine blades, different blade profiles need to be used for different parts of the blade. Figure 1 shows the blade profile distribution along the turbine blade.

This wind turbine has four blade profiles. They are distributed as shown in Figure 1 and as it can be observed, blade profile 4 is the dominating blade profile. Furthermore, it has been mentioned that each blade segment has a different contribution to the output torque.

Figure 2 shows the comparison between the lift forces in the utilized operational range [6] obtained from the different blade profiles, which is $-10^{\circ}$ to $10^{\circ}$ since high wind speed 


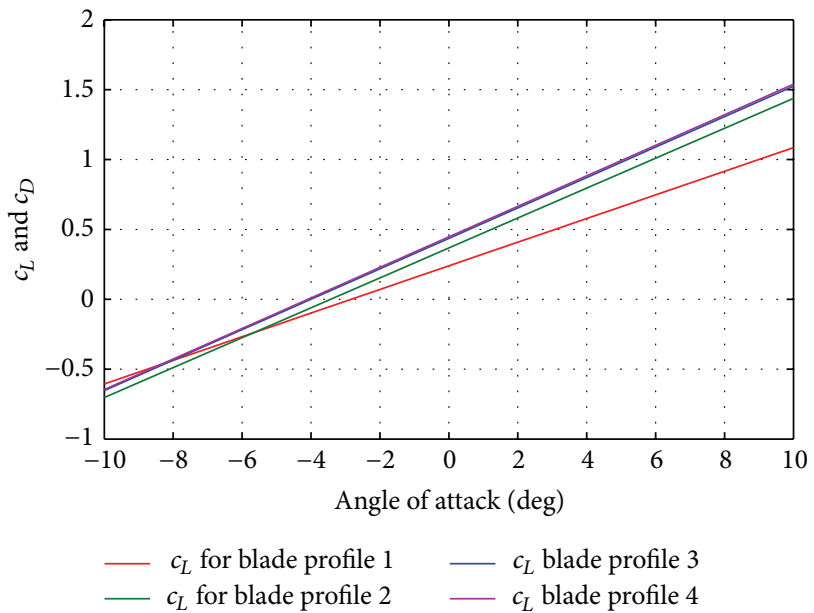

FIgURE 2: The blade profiles for the operation range of the wind turbine system. For the whole wind speed range, refer to [4].

TABLE 1: Linearized lift and drag coefficient expressions for different angle of attack regions.

\begin{tabular}{lcc}
\hline Angle of attack $(\alpha)$ & Lift coefficient $\left(c_{L}\right)$ & Drag coefficient $\left(c_{D}\right)$ \\
\hline$-10^{\circ}-10^{\circ}$ & $0.1092 * \alpha+0.4466$ & $2.23 e^{-4} * \alpha+0.0077$ \\
$10^{\circ}-18^{\circ}$ & $-0.0043 * \alpha+1.5293$ & $0.0238 * \alpha-0.2076$ \\
$18^{\circ}-28^{\circ}$ & $-0.0597 * \alpha+2.5141$ & $0.0238 * \alpha-0.2076$ \\
$28^{\circ}-45^{\circ}$ & $-1.08 e^{-4} * \alpha+0.9029$ & $0.0238 * \alpha-0.2076$ \\
$45^{\circ}-70^{\circ}$ & $-0.0164 * \alpha+1.6722$ & $0.0238 * \alpha-0.2076$ \\
\hline
\end{tabular}

region, where the pitch regulator is active, is the focus of this paper. The drag coefficients for this range are fairly close to zero. Due to the above reasons and for simplicity, blade profile 4 is used to represent the whole turbine blade.

In the whole wind speed region, for different wind speeds, it is observed that angle of attack varies in the range of $-10^{\circ}$ to $70^{\circ}$. Although the relation is non-linear in between these limits, $c_{L}$ and $c_{D}$ can be easily linearized as

$$
\begin{gathered}
c_{L i}=a_{i} \alpha+b_{i}, \\
c_{D i}=d_{i} \alpha+e_{i},
\end{gathered}
$$

where $i$ represents the number of the blade profile.

Due to the piecewise linearization, the constants $a, b, d$, and $e$ would change for each angle of attack region. Table 1 shows the expressions of $c_{L}$ and $c_{D}$ for different regions. Figure 3 shows the original and the linearized data of the lift and drag coefficients for the operating range of the angle of attack.

\section{Derivation of the Pitch Regulator}

The wind turbine considered in this paper is a variable-speed wind turbine with pitching ability. Operation of the variable speed wind turbine differs according to the wind speed. Due to the operation principles, the wind speed range is divided into three regions. These are named the low, the middle, and the high wind speed regions. Figure 4 shows the power output

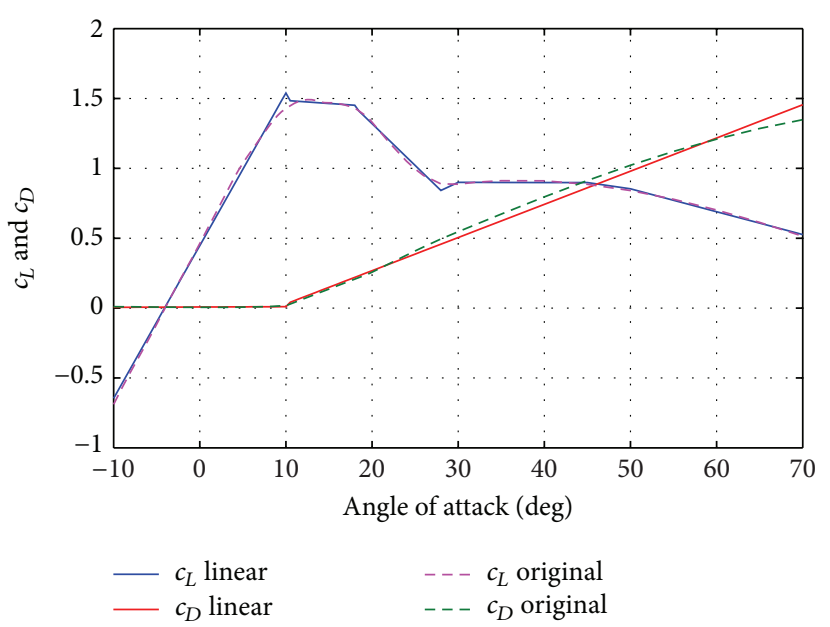

FIGURE 3: Original and linearized blade profiles. As it is observed the linearization is made piecewise and the error between the original and the linearized data is minimized.

and the rotor speed behavior of the wind turbine in the whole wind speed range. It is observed, that for the low wind speed region, the power output and the rotor speed increase with the wind speed. For the middle wind speed region, the rotor speed has reached the rated value, while the power output still increases. And lastly, for the high wind speed region, the output power reaches its rated value as well. It is desirable that both the rotor speed and the electric power are kept close to their corresponding rated values.

According to the different operation principles, different control strategies ought to be developed in order to achieve the optimal operation. The level of the captured power from the wind turbine can be easily understood from the behavior of the power coefficient, $C_{p}$. Figure 5 presents the behavior of $C_{p}$ according to the tip speed ratio, $\lambda$, and the pitch angle, $\beta$. The "optimal operation path" of the turbine is depicted as well, which is marked with the stars. It is shown in this figure that $C_{p}$ decreases with the increasing pitch angle.

It is observed from Figure 5 that the pitch angle is not changed for the low and the middle speed regions; however, it constantly increases at the high speed region. In this way, an increase in the rotor speed and the power output is prevented. Furthermore, for the low speed range, $C_{p}$ can be maximized due to the variable-speed operation.

A wind turbine controller which could maximize $C_{p}$ for the low wind speed operation, has a robust control in the middle wind speed operation and keeps the output power and the rotor speed constant for high wind speeds. A suitable design is shown in Figure 6. It is worth emphasizing that this paper focuses on the pitch controller; therefore, the only part that is explained further is the controller for the high wind speed region. For the information of the whole system, please refer to [6].

Since the study focuses on the wind turbine operation at high wind speeds, the pitching is active. In order to control the pitch angle of the wind turbine for different wind speeds, a pitch regulator is necessary. An analytically derived regulator 
TABLE 2: Different $K$ values calculated through the different blade profiles. All the $K$ values are similar to each other.

\begin{tabular}{lcccc}
\hline Wind speed & $K$ (blade profile 1) & $K$ (blade profile 2) & $K$ (blade profile 3) & $K$ (blade profile 4) \\
\hline 16 & -142150 & -182420 & -185040 & -186990 \\
17 & -152560 & -195590 & -198440 & -200430 \\
18 & -163170 & -209040 & -212120 & -214170 \\
19 & -174010 & -222760 & -226080 & -228180 \\
20 & -185080 & -236780 & -240340 & -242490 \\
21 & -196380 & -251090 & -254910 & -257100 \\
22 & -207930 & -265710 & -269780 & -272040 \\
23 & -219720 & -280650 & -284980 & -287280 \\
24 & -231770 & -295910 & -300510 & -302880 \\
25 & -244080 & -311500 & -316370 & -318780 \\
\hline
\end{tabular}

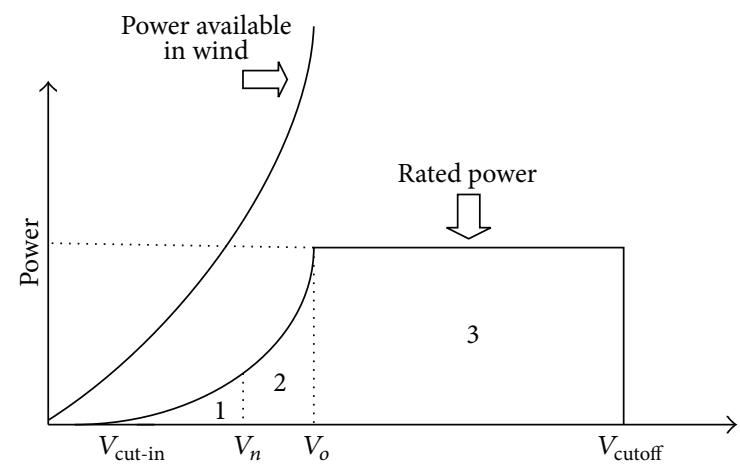

(a)

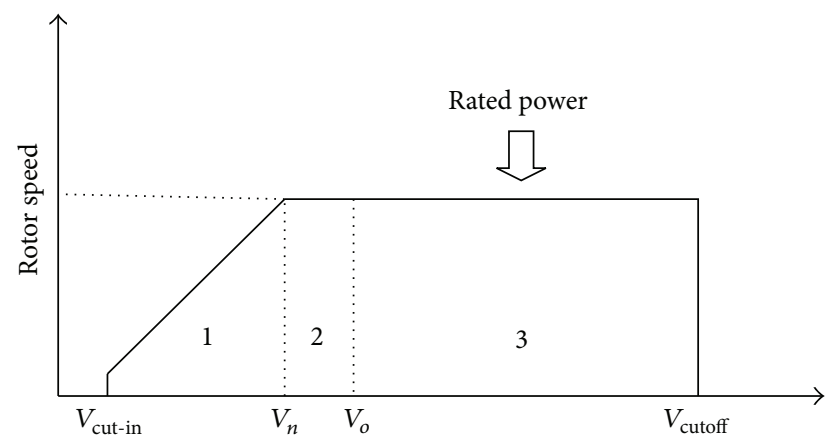

(b)

FIgURE 4: Wind turbine operation in the whole wind speed range.

would be convenient to have and for this, there preferably should be a linear relation between the pitch angle and the output torque. It is assumed that the torque is directly proportional to the pitch angle and the relation between them is

$$
T=f(\beta)=K \beta,
$$

where $\beta$ is the pitch angle and $K$ is a constant that represents the relation between pitch angle and output torque. Table 2 shows the different $K$ values obtained for different blade profiles.

The pitch regulator is designed according to this assumption using the internal mode control method [9]. Figure 7

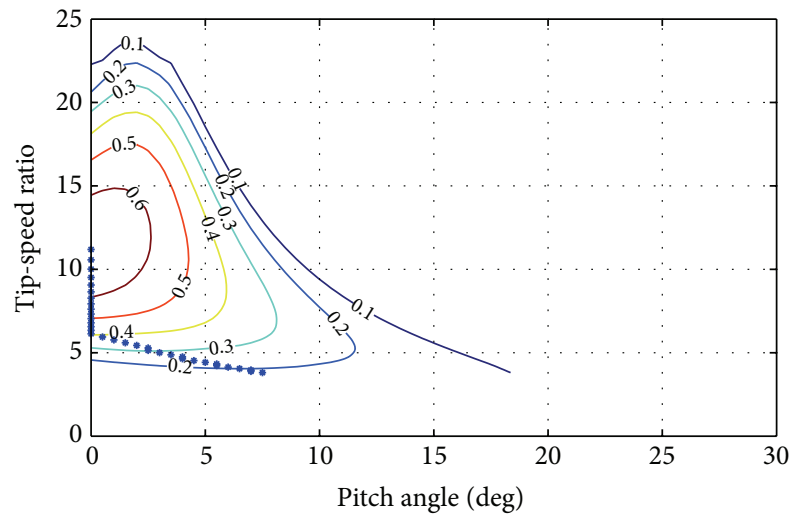

Figure 5: The contour plot shows the $C_{p}$ as a function of $\lambda$ and $\beta$ and the star indicates the optimal operation for the wind turbine.

shows the controller scheme of the pitch regulated wind turbine model.

If the pitch regulator is named $F$, then the system can be represented as

$$
\frac{\omega}{\omega^{*}}=\frac{F(K / s J)}{1+F(K / s J)} .
$$

Since the torque-pitch angle relation is assumed as in (7), the pitch regulator is designed as a P-controller. Therefore, a pitch regulator can be expressed as

$$
F=\frac{\alpha J}{K}=k_{p}
$$

where $k_{p}$ is the proportional parameter of the pitch regulator and $\alpha$ is the controller bandwidth. The next step of the controller development is to determine $K$ in terms of the turbine data and blade profile as well as the wind and rotor speed.

\section{Model Development}

The general expression of the torque contribution from a single blade segment is as in (2). Here, all the lift and drag coefficients are constant values for an individual situation of 


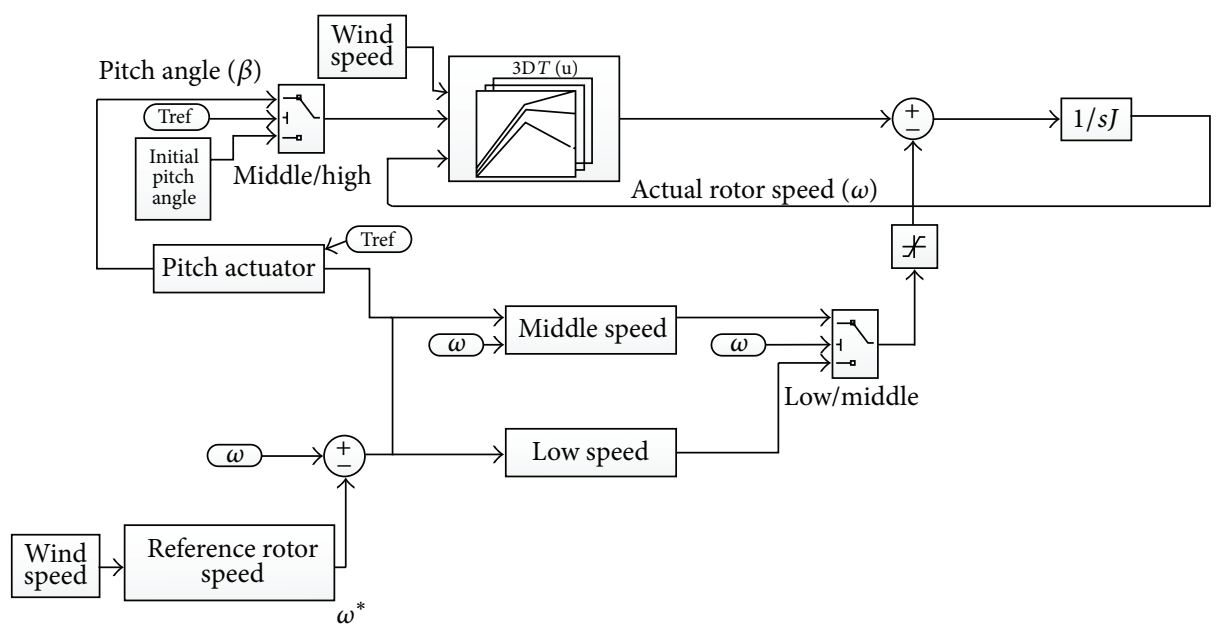

Figure 6: The controller scheme that is valid for the whole operational range.

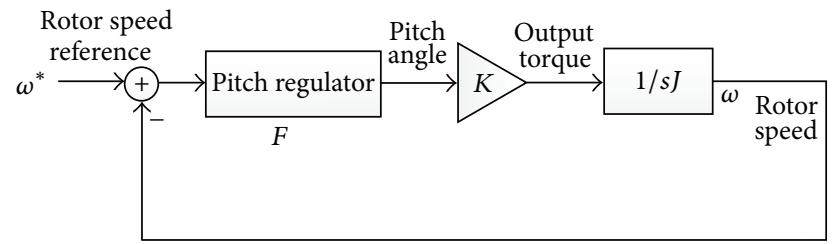

FIGURE 7: The general scheme of the pitch regulated wind turbine system.

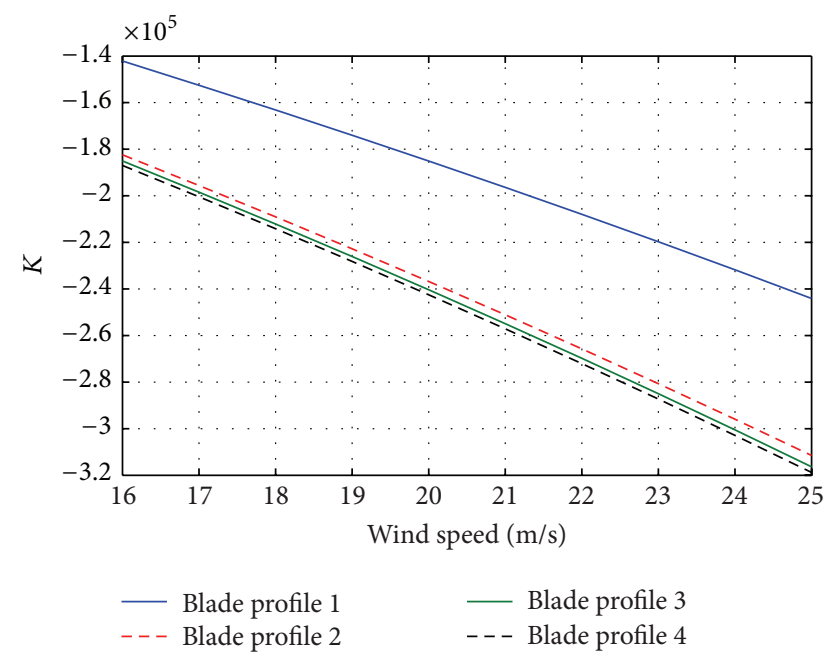

Figure 8: K parameter obtained by using different blade profiles.

the wind and the wind turbine. This means that, in order to have the output torque as a function of pitch angle, the lift and drag coefficient should be a function of it as well. Equation (6) show that $c_{L}$ and $c_{D}$ are functions of the angle of attack. The angle of attack is expressed as

$$
\alpha=\phi-\beta
$$

which means that $\alpha$ and therefore $c_{L}$ and $c_{D}$ are functions of the pitch angle.
The pitch regulated system is designed to operate in the high wind speed region. According to [6], the angle of attack in this region is between $-10^{\circ}$ and $10^{\circ}$. Table 1 shows that, for this $\alpha$ interval, the slope and the intercept values for $c_{L}$ and $c_{D}$ equations can be set to constants. The torque contribution is now expressed as

$$
\Delta T=\frac{1}{2} \rho W^{2} c \Delta r((a \alpha+b) \sin \phi-(d \alpha+e) \cos \phi) r .
$$

In the light of the explanations above, it is understood that the torque contribution of a blade segment is actually a function of the pitch angle. The relation can be expressed as the partial derivation of $\Delta T$ according to $\beta$

$$
K=\frac{\partial T}{\partial \beta}=\frac{1}{2} \rho W^{2} c \Delta r(a \sin \phi-d \cos \phi) r .
$$

This expression of $K$ is needed in order to determine the controller parameter $k_{p}$, as presented in (9). As shown in (12), $K$ is depending on the wind speed and Figure 8 shows the value of $K$ for different wind speed with different blade profiles. As a consequence of this, there is a different $k_{p}$ parameter for each wind speed. However, due to practicality, one single value will be chosen to be used for the whole operating range, which is the value obtained at a $20 \mathrm{~m} / \mathrm{s}$ wind speed. Furthermore, blade profile 4 is considered to be the only profile for the whole turbine blade. While blade profiles 2 and 3 give quite similar results to the ones that are obtained by using blade profile 4 , blade profile 1 contributes to an approximately $20 \%$ of error. Fortunately, the part that has blade profile 1 contributes very little to the output torque [6]; therefore, considering blade profile 4 as the sole profile for the turbine does not affect the overall torque output of the system, significantly.

Another important point regarding the pitch regulator design is that the output torque expression is more complicated than that assumed in (7). Although the torque-pitch relation still can be expressed as a first-order function, there is an offset. This offset that varies with wind speed and the blade segment would accumulate into a large steady state error, if 


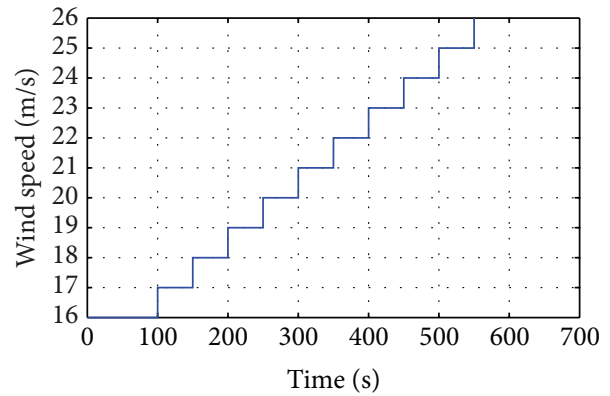

(a)

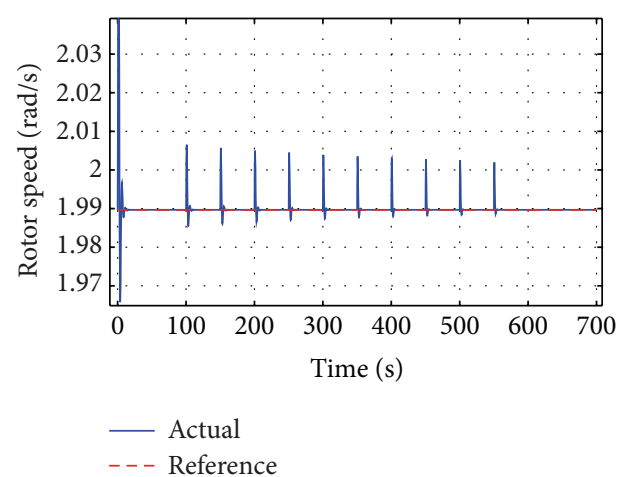

(c)

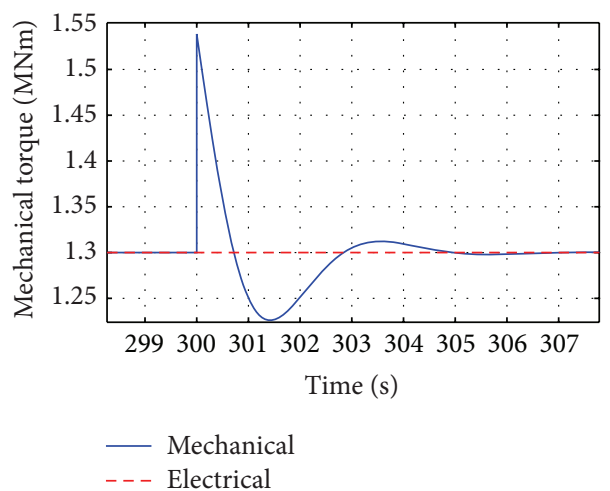

(e)

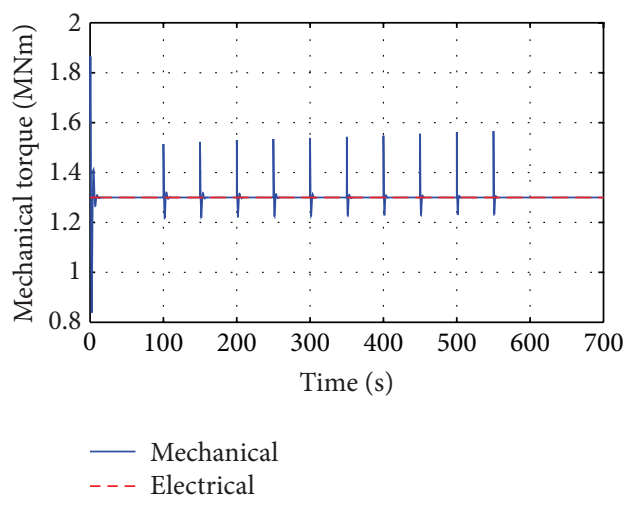

(b)

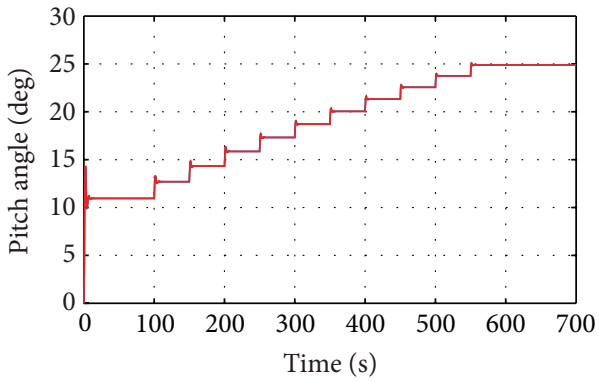

(d)

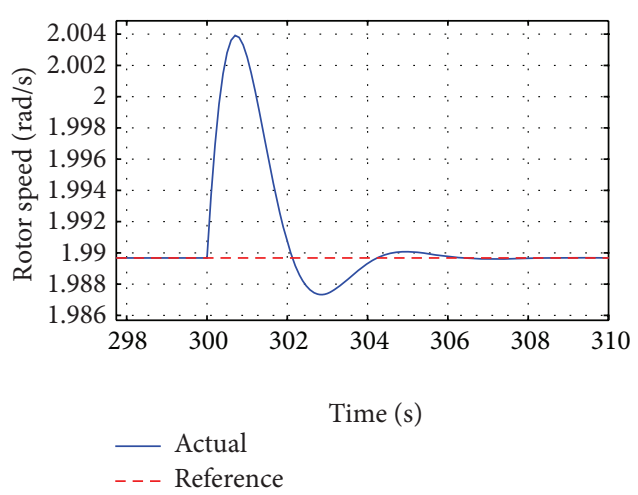

(f)

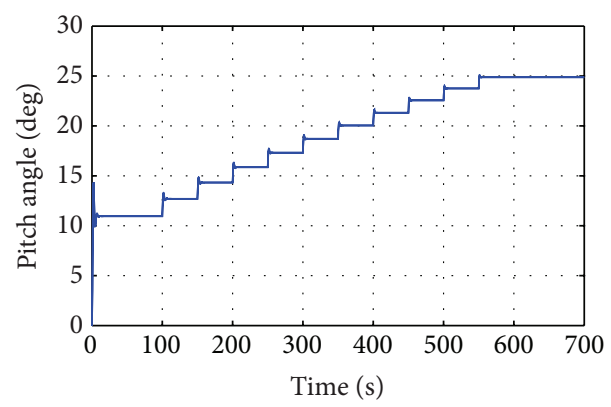

(g)

FIGURE 9: Step response of the pitch regulated WT system under ideal grid conditions: (a) wind speed, (b) output torque, (c) rotor speed, (d) pitch angle for the whole high speed region and (e) output torque, $(\mathrm{f})$ rotor speed, and (g) pitch angle for one wind step. 


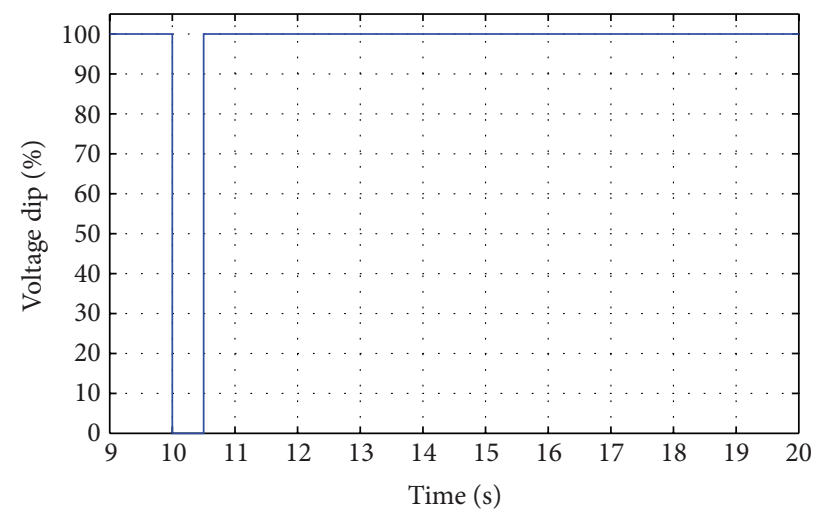

(a)

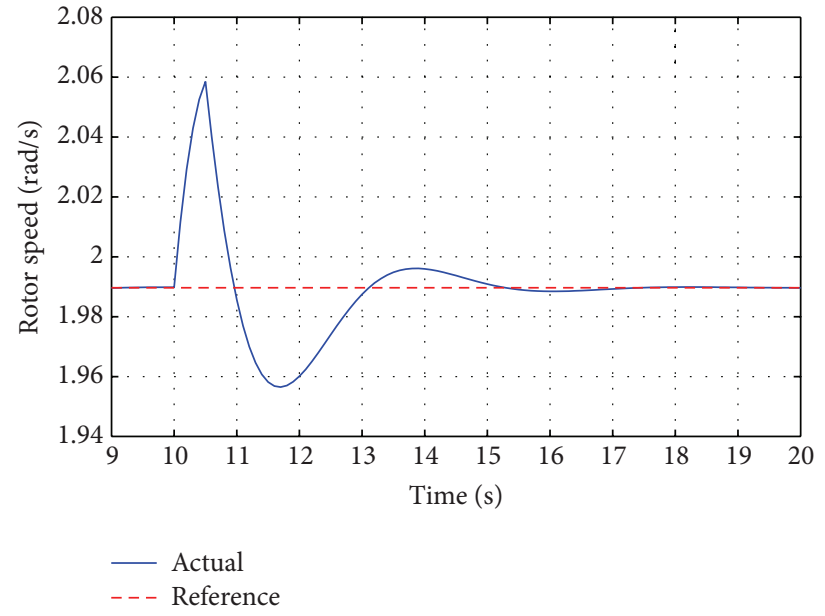

(c)

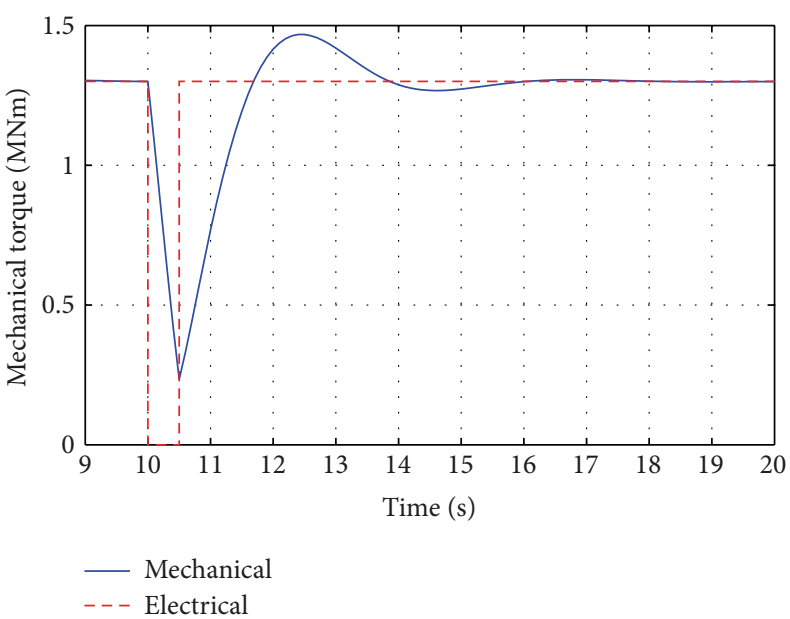

(b)

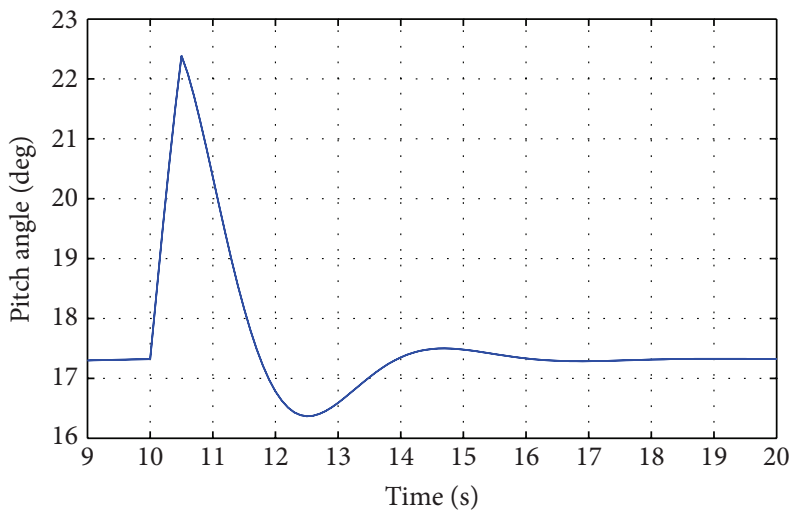

(d)

Figure 10: Step response of the pitch regulated WT system under $100 \%$ voltage dip: (a) power level, (b) output torque, (c) rotor speed, and (d) pitch angle.

not taken into consideration. Therefore, in order to prevent a large steady state error, the pitch controller needs to have an integral part in addition to the proportional part.

The integral parameter of the pitch regulator, in the absence of a natural damping, is calculated through the active damping method [10] as

$$
k_{i}=\frac{\alpha^{2} J}{K} .
$$

The analytically expressed pitch regulated speed controller of the wind turbine system is hence derived.

\section{Investigating the Performance of the Pitch Regulator}

6.1. Verifying Normal Operation Behavior. The pitch regulated WT system is now studied when operating in the high speed region, which means that the output torque and the rotor speed of the turbine are operating close to their rated values. The nominal rotor speed of the wind turbine is $19 \mathrm{rpm}$. The nominal output power that this turbine can provide is
$2 \mathrm{MW}$. Firstly, the response of the pitch regulated system is simulated for the wind increases. While the WT operates in steady state, the wind speed would be changing stepwise, and each step is initiated 50 seconds after the previous value. The results obtained from these conditions are shown in Figure 9.

When the WT system is subjected to a step wise increase in the wind speed, the shaft torque and rotor speed have slight overshoots. This is due to the integral part of the pitch regulator, which has been calculated through the active damping method. Although the instantaneous wind speed increase is slightly unrealistic, the overshoot of the rotor speed is $0.7 \%$, which is an acceptable value, due to wind turbine regulations. It is worth noting that the wind steps simulated in this paper are instantaneous, which is a very drastic event compared to the realistic winds. The increase in the shaft torque is balanced by the pitching action. The pitch angle is increased in order to keep the shaft torque constant at the rated value for the wind turbine.

6.2. Voltage Dip. According to IEEE 1159, a reduction in the voltage is considered as a voltage dip when the RMS voltage 


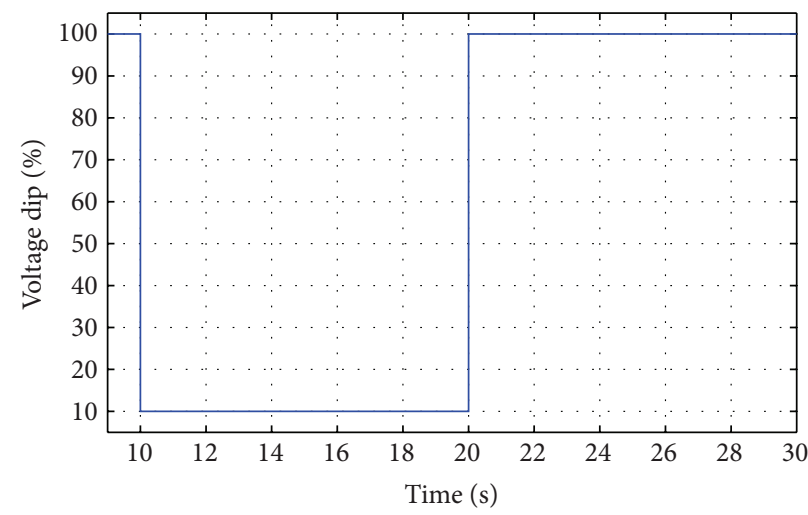

(a)

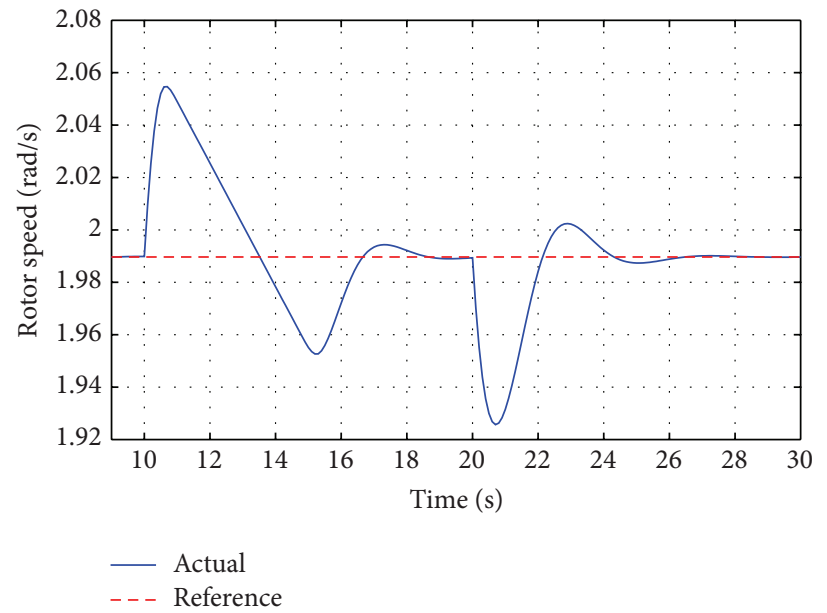

(c)

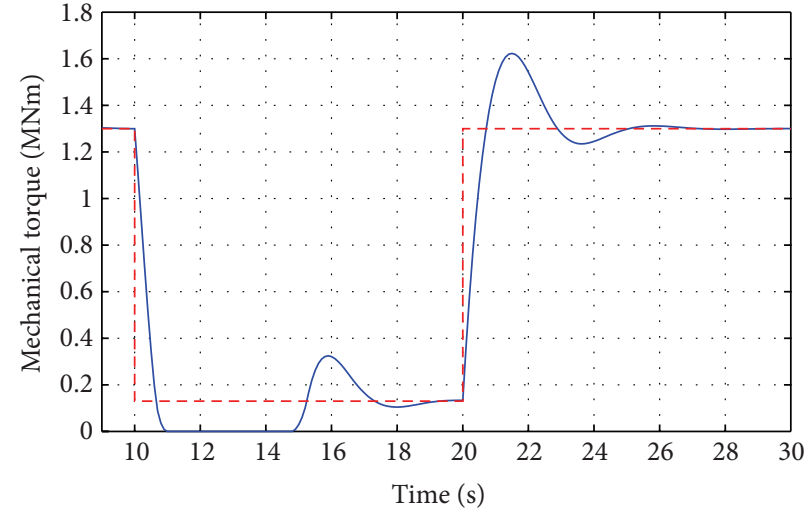

(b)

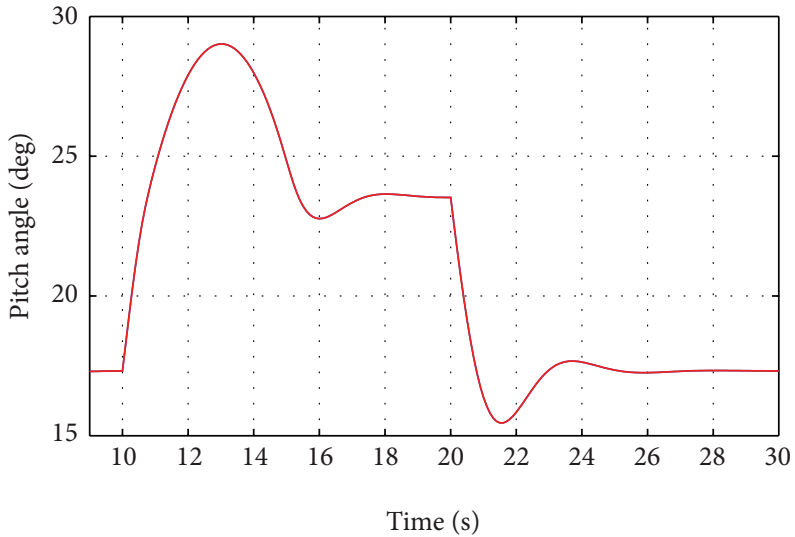

(d)

FIGURE 11: Step response of the pitch regulated WT system when the spinning reserve ability is activated and the power is reduced with $90 \%$ : (a) power level, (b) output torque, and (c) rotor speed, (d) pitch angle.

decreases between 0.1 and 0.9 p.u of the nominal voltage for a duration of half a cycle up to one minute [11].

Voltage dips are undesired incidents and might cause disturbances for the wind turbine system. There are several solutions for protecting the WT from the DC-link overvoltages that occur during a voltage dip and still keeping them online as stated by the grid codes, one of which is to use a braking chopper. A braking chopper is a device that discharges the DC-link of the wind turbine during a voltage dip and in this way prevents the DC-link voltage from being too high. When a braking chopper is used, the turbine operates undisturbedly, since all the excess energy is dissipated in the braking chopper. However, in this paper, pitch regulation is used in order to cope with the problem that all energies cannot be sent to the grid during the dip.

The system is tested for the most severe case, when the system experiences a voltage dip with $0 \%$ remaining voltage. The duration of the voltage dip is $500 \mathrm{~ms}$. The simulation results are presented in Figure 10. When the dip occurs, the reference torque directly decreases to 0 from its prefault level. Generally, at a full power converter system, the wind turbine is not affected by the grid disturbances directly; however, in order to be able to control the WT system with the pitch regulator, the reference torque must be adjusted likewise. The rotor speed of the wind turbine accelerates and results in a $3.5 \%$ overshoot.

In order to be able to reduce the shaft torque by $100 \%$, the pitch angle increases to $5^{\circ}$. This is expected due to the significant reduction in the voltage level. The effect of the pitch actuator is ignored in this paper, for simplicity.

All the parameters have overshoots but since the voltage dip is a short duration disturbance, the time to reach the steady state is not affected. As noted from Figure 10, the pitch regulated WT system is able to operate under severe voltage dips, up to $100 \%$ reduction.

6.3. Spinning Reserve Ability. A loss of load or generation in a power system leads to a frequency disturbance. These disturbances can be mitigated to a slight degree by changing the power level of wind turbines connected to the power system. Therefore, the pitch regulated WT system developed in this paper should be able to operate when the power needed by the system changes. 
Of course, mainly the case with a required power increase is of the greatest interest; however, in order to broaden the investigation, the power level is first reduced and then increased back to its nominal level. The instant increase of the power level will show the spinning reserve ability of the pitch regulated WT system.

The spinning reserve action in this paper is simulated as the severe case scenario and for a longer duration than the voltage dip. The power level is decreased to $10 \%$ and the duration of it is 10 seconds. The simulation results can be observed from Figure 11.

When the power level is reduced, the reference torque directly decreases by $90 \%$, instantly. Due to the insufficient electric loading of the generator for the present operating conditions, the rotor speed starts to increase. The rotor speed increase is detected by the controller and it is pushed back to its rated level, having a $3 \%$ overshoot when the controlling process starts.

The pitch angle difference adjusts the torque behavior of the WT system, and to be able to reduce the output torque by $90 \%$, the pitch angle increases from its prefault value, $17.3^{\circ}$ to $28^{\circ}$ at first, but reaches a steady-state value of $23^{\circ}$ for the new operation level. After the power is restored, the pitch angle decreases to its prefault value.

When the power level is restored, the rotor speed is decreased due to the increased electrical load; however, it reaches steady state shortly. From Figure 11 it can be observed that the pitch regulated WT system is able to operate when there is a drastic change in the power level, both for an increase and for a reduction.

\section{Conclusion}

A pitch regulator for a wind turbine system expressed in analytical terms has been designed on the basis of a simplified aerodynamic model of the wind turbine. These simplifications are made in order to calculate the torque produced by the wind turbine in an easily described way. This modified torque calculation allows us to extract a linear, torque-pitch angle relation, which is used while deriving the pitch regulator. Moreover, one single setting of the regulator parameters, which have been obtained using blade profile 4 at $20 \mathrm{~m} / \mathrm{s}$, is shown to be sufficient for the whole operating region.

The procedure presented in this paper is also applicable to other turbines. Using the aerodynamic data of that wind turbine, a pitch regulator can easily be derived.

The operation of the pitch regulated wind turbine system is shown to be robust and stable according to the simulation results. Since the offset of the pitch angle-mechanical torque relation is neglected while designing the regulator, the excess part of the equation is balanced by deriving an integrator, according to the "active damping" method. The integrator constant calculated through this method results in overshoots of the controlled parameters. However, since these overshoots are in the $1 \%$ range, accordingly the performance of the system is almost not affected. If desired, the integrator can be enhanced in order to have a smoother control.
The pitch regulated wind turbine system is able to operate even when a $100 \%$ voltage dip occurs. Furthermore, the system is tested for the spinning reserve ability and the system can at least handle a $90 \%$ change in the power level. After both disturbances, the pitch regulated WT system can easily return to steady state operation. The system is able to satisfy the grid codes which are explained in [12].

\section{References}

[1] E. Muljadi, "Pitch-controlled variable-speed wind turbine generation," IEEE Transactions on Industry Applications, vol. 37, no. 1, pp. 240-246, 2001.

[2] S. H. Lee, Y. J. Joo, J. Back, and J. H. Seo, "Sliding mode controller for torque and pitch control of wind power system based on PMSG," in International Conference on Control, Automation and Systems (ICCAS '10), pp. 1079-1084, October 2010.

[3] J. Jonkman, S. Butterfield, W. Musial, and G. Scott, "Definition of a \% MW reference wind turbine for offshore system development," Tech. Rep. NREL/TP-500-38060, National Renewable energy Laboratory, February 2009.

[4] F. Bianchi, H. De Battista, and R. J. Mantz, Wind Turbine Control Systems-Principles, Modelling and Gain Scheduling Design, Springer, London, UK, 2007.

[5] D. Eggleston and F. Stoddard, Wind Turbine Engineering Design, Van Nostrand Reinhold, 1987.

[6] P. Tokat, Development and Evaluation of a Pitch Regulator for a Variable Speed Wind Turbine, Chalmers University of Technology, March 2012.

[7] S. Heier, Wind Energy Conversion Systems, John Wiley \& Sons, 2006.

[8] G. Ingram, Wind Turbine Blade Analysis Using the Blade Element Momentum Method, Durham University, 2011.

[9] T. Thiringer and A. Petersson, Control of a Variable-Speed PitchRegulated Wind Turbine, Chalmers University of Technology, 2005.

[10] L. Harnefors, Control of Variable-Speed Drives, Mälardalen University, 2002.

[11] IEEE Standard 1159-2009 (Revision of IEEE Standard 11591995), "IEEE Recommended Practica for Monitoring Electric Power Quality".

[12] M. Tsili and S. Papathanassiou, "A review of grid code technical requirements for wind farms," IET Renewable Power Generation, vol. 3, no. 3, pp. 308-332, 2009. 


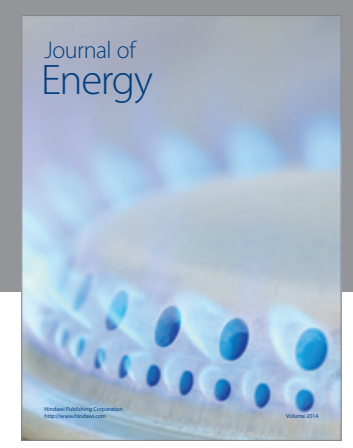

Journal of

Industrial Engineering
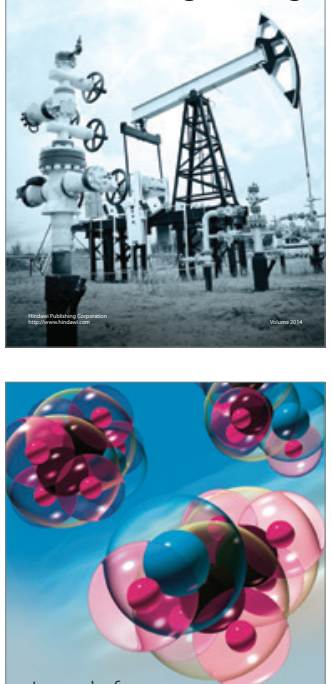

Fuels
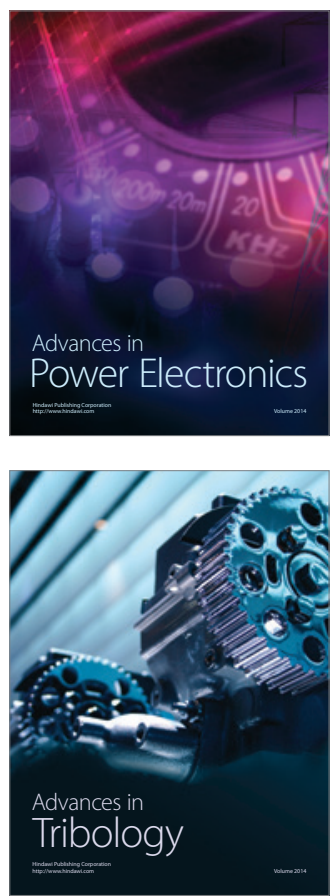

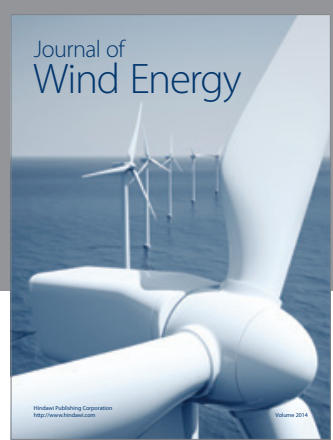

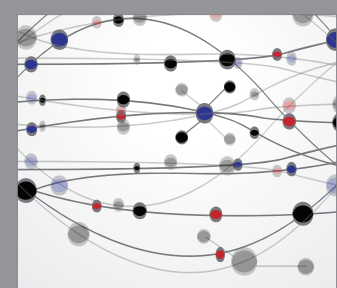

The Scientific World Journal

Submit your manuscripts at http://www.hindawi.com

Journal of

Structures
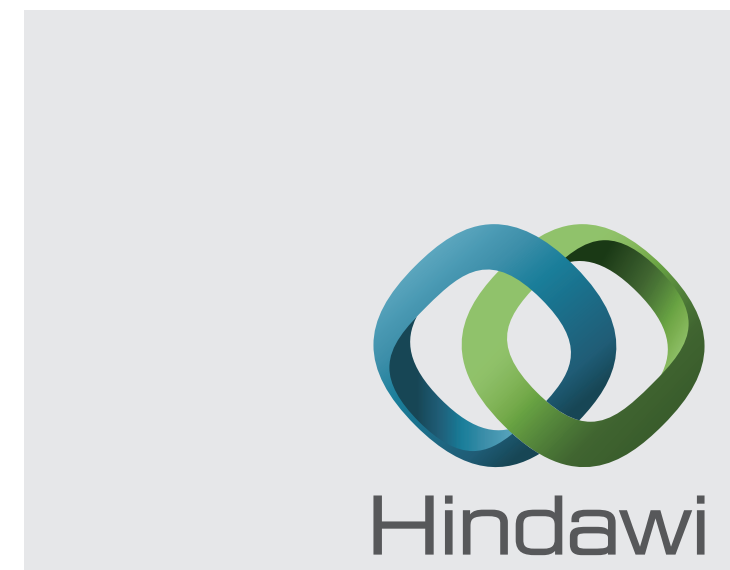

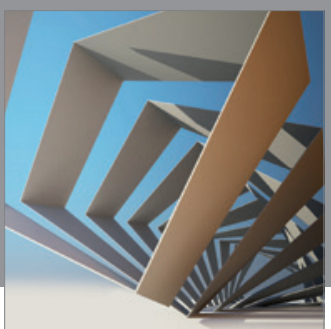

Rotating

Machinery
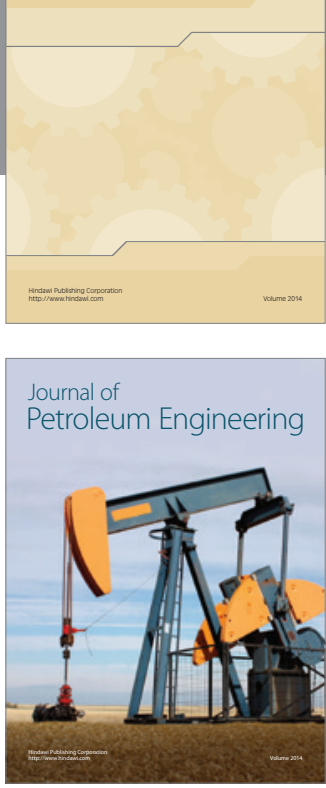

Journal of

Solar Energy
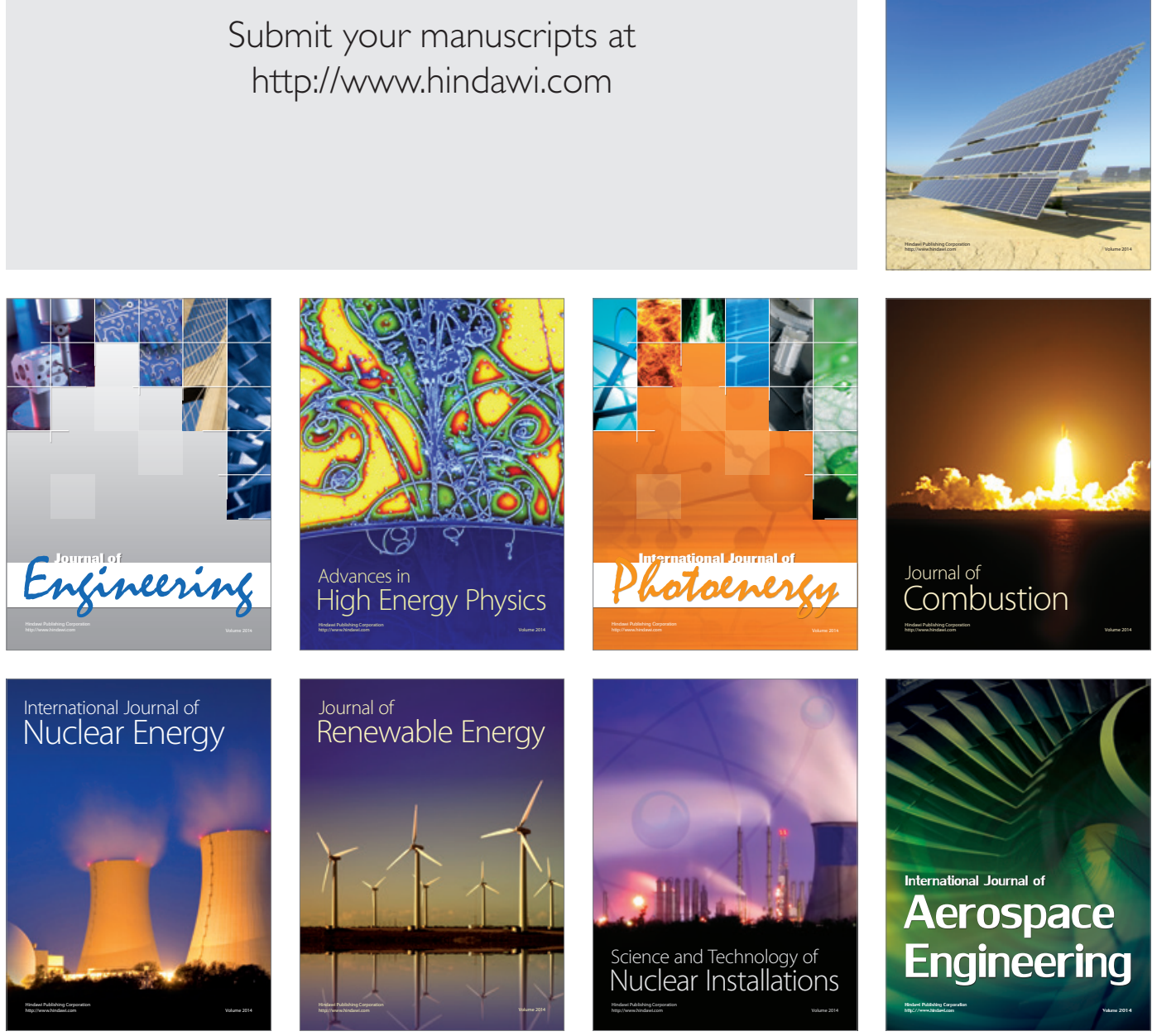